\title{
Ação de produtos naturais sobre a sobrevivência de Argyrotaenia sphaleropa (Meyrick) (Lepidoptera: Tortricidae) e seletividade de inseticidas utilizados na produção orgânica de videira sobre Trichogramma pretiosum Riley (Hymenoptera: Trichogrammatidae)
}

\author{
Action of natural products on the survival of Argyrotaenia sphaleropa (Meyrick) (Lepidoptera: Tortricidae) \\ and selectivity of insecticides used in the organic production of vine on Trichogramma pretiosum Riley \\ (Hymenoptera: Trichogrammatidae)
}

\author{
Wilson José Morandi Filho ${ }^{1}$ Marcos Botton ${ }^{2}$ Anderson Dionei Grützmacher ${ }^{3}$ \\ Fabrizio Pinheiro Giolo ${ }^{1}$ Cristiane Gindri Manzoni ${ }^{1}$
}

\section{RESUMO}

Nesse trabalho, foi estudado o efeito de formulações comerciais de inseticidas, com ênfase para os produtos permitidos na produção orgânica (nim, piretro natural e extrato pirolenhoso) para o controle de Argyrotaenia sphaleropa (Meyrick) (Lepidoptera: Tortricidae) e sua atuação sobre o parasitóide de ovos Trichogramma pretiosum Riley (Hymenoptera: Trichogrammatidae), em laboratório. Os inseticidas Natuneem ${ }^{\circledR}(1500 \mathrm{ppm}$ de Azadirachta indica por litro) e o extrato pirolenhoso (Biopirol $7 \mathrm{M}^{\circledR}$ ), nas dosagens de 250 e $500 \mathrm{~mL} 100 \mathrm{~L}^{-1}$, não foram eficientes no controle de $\mathbf{A}$. sphaleropa quando aplicados sobre folhas de videira (Vitis sp.) cultivar "Chardonnay", enquanto que o piretro natural (250 e 500mL $\left.100 \mathrm{~L}^{-1}\right)$ resultou em mortalidade significativa de 77,65 e 85,88\% dos insetos, respectivamente, 120 horas após a aplicação. $O$ efeito secundário foi avaliado sobre adultos do parasitóide de ovos T. pretiosum, seguindo a metodologia da International Organization for Biological and Integrated Control (IOBC). Os inseticidas Natuneem ${ }^{\circledR}\left(500 \mathrm{~mL} 100 \mathrm{~L}^{-1}\right)$,

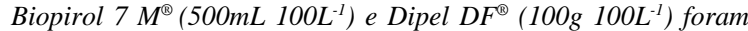
inócuos ( $<30 \%$ de redução no parasitismo por T. pretiosum), enquanto o piretro natural, nas dosagens de $250 \mathrm{~mL} 100 \mathrm{~L}^{-1}$ e $500 \mathrm{~mL} 100 \mathrm{~L}^{-1}$, foi classificado como moderadamente nocivo (80-99\% de redução no parasitismo) e nocivo (>99\% de redução no parasitismo), respectivamente, equivalendo-se ao efeito do fosforado Lebaycid $500^{\circledR}\left(100 \mathrm{~mL} 100 \mathrm{~L}^{-1}\right)$.

Palavras-chave: inseticidas orgânicos, uva, efeito secundário, parasitóide de ovos.

\begin{abstract}
This work was conducted to study the effect of commercial formulations of insecticides with emphasis on that allowed in the organic production (neem, natural piretro and pirolenhoso extract) to control Argyrotaenia sphaleropa (Meyrick) (Lepidoptera: Tortricidae) and their performance on the egg parasitoid Trichogramma pretiosum Riley (Hymenoptera: Trichogrammatidae) adults. The insecticides Natuneem ${ }^{\circledR}\left(1500 p p m\right.$ of azadirachtin $\left.L^{-1}\right)$ and the pirolenhoso extract (Biopirol $7 \mathrm{M}^{\circledR}$ ) (250 and 500mL 100L-1) were not efficient in the control of A. sphaleropa when applied over grapevine leaves (Vitis sp.) cultivate Chardonnay. Natural

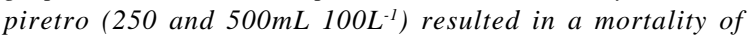
77.65 and $85.88 \%$ of insects, respectively 120 hours after application. The secondary effect of insecticides allowed was evaluated on adults of the egg parasitoid T. pretiosum following International Organization for Biological and Integrated Control (IOBC) methodology. It was observed that Natuneem ${ }^{\circledR}$ (500mL 100L-1), Biopirol $7 \mathrm{M}^{\circledR}\left(500 \mathrm{~mL} 100 \mathrm{~L}^{-1}\right)$ and Dipel DF ${ }^{\circledR}$

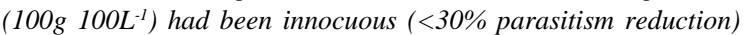
while natural piretro $\left(250 \mathrm{~mL} 100 \mathrm{~L}^{-1}\right)$ was classified as moderately harmful (80-99\% of parasitism reduction). Natural piretro in the higher dose $\left(500 \mathrm{~mL}_{\left.100 \mathrm{~L}^{-1}\right)}\right.$ showed a parasitism reduction in more than $99 \%$ in the same way that the pattern Lebaycid $500^{\circledR}\left(100 \mathrm{~mL} 100 \mathrm{~L}^{-1}\right)$.
\end{abstract}

Key words: organic insecticides, grape, secondary effects, egg parasitoid.

\footnotetext{
${ }^{1}$ Programa de Pós-graduação em Fitossanidade, Departamento de Fitossanidade, Faculdade de Agronomia Eliseu Maciel (FAEM), Universidade Federal de Pelotas (UFPel). Campus Universitário s/n, CP 354, 96.010-900, Pelotas, RS, Brasil. E-mail: wilsonmorandi@yahoo.com.br, fgiolo.faem@ufpel.tche.br, cristianemanzoni@hotmail.com.

${ }^{2}$ Embrapa Uva e Vinho. Rua Livramento 515, CP 130, 95.700-000, Bento Gonçalves, RS, Brasil. E-mail: marcos@cnpuv.embrapa.br. ${ }^{3}$ Departamento de Fitossanidade, FAEM, UFPel, Pelotas, RS, Brasil. E-mail: adgrutzm@ufpel.tche.br. Autor para correspondência.
} 


\section{INTRODUÇÃO}

Argyrotaenia sphaleropa (Meyrick) (Lepidoptera: Tortricidae) é uma espécie nativa da América do Sul, ocorrendo no Uruguai (BENTANCOURT \& SCATONI, 1995), Argentina (KÖHLER, 1939), Bolívia, Peru (MEYRICK, 1909) e Brasil (BIEZANKO, 1961). É um inseto polífago, constituindo-se em importante praga de frutíferas temperadas no Uruguai (NUÑEZ et al., 2002) e no Brasil, onde danifica o caquizeiro (BAVARESCO, 2004), a pereira (NORA \& SUGIURA, 2001), o pessegueiro (BOTTON et al., 2003a) e a videira (BIEZANKO, 1961; BOTTON et al., 2003b). Especificamente na videira, esta espécie provoca o rompimento das bagas, resultando no extravasamento do suco sobre o qual proliferam bactérias que provocam a podridão ácida, reduzindo a qualidade dos vinhos ou depreciando os cachos para o comércio in natura (BOTTON et al., 2003b).

Poucas informações estão disponíveis em relação ao controle dessa praga nas diferentes culturas que não seja o uso exclusivo de inseticidas químicos (BAVARESCO, 2004). Porém, existe uma demanda crescente por métodos alternativos, visando a substituir o controle químico, especialmente quando as fruteiras são cultivadas no sistema orgânico de produção (MARTINEZ, 2002).

Alternativas para o controle de pragas na produção orgânica dizem respeito ao uso de plantas inseticidas, com destaque para o nim Azadirachta indica (Meliaceae) (SCHMUTTERER, 1990; MARTINEZ, 2002), o piretro natural extraído das flores do Chrysanthemum cinerariaefolium (Compositae) (TAMM et al., 2004), além do emprego da bactéria Bacillus thuringiensis (MORANDI FILHO et al., 2004).

Outro composto que tem sido comercializado para o controle de pragas na produção orgânica é o extrato pirolenhoso, produto obtido por meio da condensação da fumaça produzida durante a carbonização da madeira (ENCARNAÇÃO, 2001) e que, em hipótese, apresenta propriedades inseticidas (MIYASAKA et al., 1999). O extrato pirolenhoso tem sido pesquisado e utilizado principalmente no Japão, onde é empregado como fertilizante, inferindo-se que o mesmo também atua no controle de pragas e doenças (TSUZUKI et al., 2000).

Os inseticidas naturais normalmente não apresentam problemas de contaminação ambiental, resíduos nos alimentos, efeitos prejudiciais a organismos benéficos e seleção de insetos resistentes (SCHMUTTERER, 1990). Entretanto, vários autores têm demonstrado uma resposta diferenciada dos inimigos naturais em função da aplicação de tais inseticidas, levando à necessidade de se gerar informações específicas para cada formulação, inimigo natural e sistema de produção (KLEMM \& SCHMUTTERER, 1993; GONÇALVES-GERVÁSIO \& VENDRAMIM, 2004).

Dentre os agentes de controle biológico de insetos-praga, os parasitóides de ovos do gênero Trichogramma possuem distribuição mundial, estando associados a várias espécies hospedeiras, inclusive já tendo sido relatado parasitando ovos de $\boldsymbol{A}$. sphaleropa na cultura da videira (BASSO et al., 1999). Além disso, esses parasitóides foram escolhidos para serem uma das espécies-padrão, recomendadas na União Européia pela "International Organization for Biological and Integrated Control of Noxious Animals and Plants (IOBC), West Palearctic Regional Section (WPRS)”, para avaliar o efeito de pesticidas sobre inimigos naturais quando do registro de novas moléculas.

Nesse sentido, considerando que a associação entre inimigos naturais e o emprego de produtos naturais ou inseticidas microbianos pode se constituir numa alternativa viável, para o manejo de pragas na cultura da videira, principalmente em sistema orgânico de produção, objetivou-se avaliar o efeito de formulações comerciais à base de óleo de nim, piretro natural e extrato pirolenhoso sobre lagartas de $\boldsymbol{A}$. sphaleropa, em laboratório, e conhecer o efeito desses três produtos e de $\boldsymbol{B}$. thuringiensis (Dipel $\mathrm{DF}^{\circledR}$ ) sobre adultos do parasitóide de ovos Trichogramma pretiosum Riley, em laboratório.

\section{MATERIAL E MÉTODOS}

Obtenção e criação dos insetos e local de condução dos experimentos

Os insetos utilizados nos experimentos foram obtidos da criação de $\boldsymbol{A}$. sphaleropa mantida no Laboratório de Entomologia da Embrapa Uva e Vinho $\left(25 \pm 1^{\circ} \mathrm{C}\right.$, umidade relativa de $70 \pm 10 \%$ e fotofase de 14 horas), em dieta artificial (MANFREDI-COIMBRA et al., 2005). O experimento de seletividade foi conduzido no laboratório de Biologia de Insetos, Controle Biológico e de Pesticidas e Drogas do Departamento de Fitossanidade (DFs)e da Faculdade de Agronomia “Eliseu Maciel” (FAEM), na Universidade Federal de Pelotas (UFPel), em Pelotas, RS, na temperatura de $25 \pm 1^{\circ} \mathrm{C}$, UR $70 \pm 10 \%$ e fotofase de 14 horas.

Bioensaios de ingestão em folhas de videira

Discos de folhas com seis centímetros de diâmetro da cultivar "Chardonnay" foram imersos por 15 segundos em calda inseticida contendo os respectivos tratamentos (Tabela 1). Em seguida, os 
Tabela 1 - Inseticidas avaliados para o controle de Argyrotaenia sphaleropa em laboratório e em testes de seletividade sobre adultos de Trichogramma pretiosum.

\begin{tabular}{|c|c|c|c|c|}
\hline Produto & Nome comercial & Concentração ia (ppm) & Empresa fabricante & Dosagem $\left(\mathrm{mL} 100 \mathrm{~L}^{-1}\right)$ \\
\hline $\begin{array}{l}\text { Óleo de Nim } \\
\text { (Azadirachta indica) }\end{array}$ & Natuneem $^{\circledR}$ & 1500 & Natural Rural & 250 \\
\hline $\begin{array}{l}\text { Óleo de Nim } \\
\text { (Azadirachta indica) }\end{array}$ & Natuneem ${ }^{\circledR}$ & 1500 & Natural Rural & 500 \\
\hline Bacillus thuringiensis & Dipel DF ${ }^{\circledR}$ & ----- & Iharabras & 100 \\
\hline Piretro Natural & A definir & N.I ${ }^{1}$ & BioControle & 250 \\
\hline Piretro Natural & A definir & N.I & BioControle & 500 \\
\hline Extrato Pirolenhoso & Biopirol $7 \mathrm{M}^{\circledR}$ & ---- & Biocarbo & 250 \\
\hline Extrato Pirolenhoso & Biopirol $7 \mathrm{M}^{\circledR}$ & ----- & Biocarbo & 500 \\
\hline Fenthion $^{2}$ & Lebaycid $500^{\circledR}$ & 500 & Bayer & 100 \\
\hline Água destilada & Testemunha & ----- & ----- & \\
\hline
\end{tabular}

${ }^{1}$ Concentração não informada pela empresa fabricante por ser importado e estar em fase de pesquisa.

${ }^{2}$ Usado como inseticida padrão no experimento de seletividade.

discos foram secados à sombra por duas horas e transferidos em número de três para o interior de potes plásticos (5,5cm de largura e $8,5 \mathrm{~cm}$ de diâmetro), recebendo nove lagartas de $\boldsymbol{A}$. sphaleropa medindo aproximadamente 1,0cm (3 instar). O experimento foi conduzido em delineamento inteiramente casualizado, sendo que, para cada tratamento, utilizou-se dez repetições, representada cada uma por um pote plástico. O número de lagartas vivas foi avaliado 24, 48, 72, 96 e 120 horas após aplicação (HAA) dos produtos, sendo a mortalidade corrigida pela fórmula de Abbott (ABBOTT, 1925). Os resultados obtidos foram submetidos à análise de variância e as médias dos tratamentos comparadas pelo teste de Tukey ao nível de $5 \%$ de probabilidade.

Efeito de inseticidas sobre Trichogramma pretiosum

A medodologia de avaliação seguiu a preconizada pela IOBC/WPRS para testes de seletividade com parasitóides do gênero Trichogramma (HASSAN et al., 2000). A aplicação dos inseticidas foi realizada com pulverizador manual de $580 \mathrm{~mL}$ da marca Guarany ${ }^{\circledR}$, proporcionando um

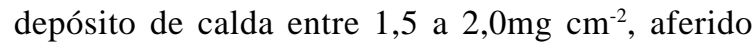
mediante balança eletrônica de precisão.

Os parasitóides $\boldsymbol{T}$. pretiosum utilizados nos experimentos foram oriundos de criação em laboratório, em câmaras climatizadas (temperatura $25 \pm 1^{\circ} \mathrm{C}$, UR $70 \pm 10 \%$ e fotofase 14 horas), utilizando-se ovos do hospedeiro alternativo Anagasta kuehniella (Zeller)
(Lepidoptera: Pyralidae) inviabilizados em lâmpada germicida.

Os testes de toxicidade foram conduzidos expondo-se os adultos (estágio mais sensível) de $\boldsymbol{T}$. pretiosum a resíduos secos dos pesticidas (Tabela 1) pulverizados sobre placas de vidro (2mm de espessura e tamanho de $13 \mathrm{~cm} \times 13 \mathrm{~cm}$ ), permanecendo estas após a pulverização à sombra por três horas para secagem da calda, formando uma película seca do produto-teste.

Tubos de emergência contendo adultos de T. pretiosum com aproximadamente 24 horas de idade foram conectados às gaiolas de exposição seis horas após a pulverização, permitindo a entrada dos insetos no interior da gaiola. Cada tubo de emergência foi constituído por uma ampola de vidro transparente de $120 \mathrm{~mm}$ de comprimento por $20 \mathrm{~mm}$ de diâmetro em uma das extremidades e de $7 \mathrm{~mm}$ na outra, contendo no seu interior um círculo de $1 \mathrm{~cm}$ de diâmetro com $250 \pm 50$ ovos previamente parasitados, aderido a uma tira de papel de $80 \mathrm{~mm}$ de comprimento por $15 \mathrm{~mm}$ de largura, com três finos filetes de alimento ( $3 \mathrm{~g}$ de gelatina, $100 \mathrm{~mL}$ de água e $200 \mathrm{~g}$ de mel). Após 24 horas da pulverização,

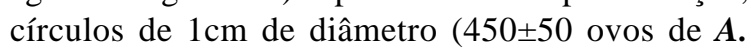
kuehniella) foram ofertados no segundo (nove círculos), terceiro (seis círculos) e quinto (três círculos) dias após pulverização, para serem parasitados, permitindo a avaliação do parasitismo e conseqüente efeito dos tratamentos.

A partir dos resultados número de ovos parasitados e número de fêmeas no interior da gaiola, obteve-se o número médio de ovos parasitados por 
fêmea de T. pretiosum para cada tratamento. As reduções no parasitismo dos pesticidas-teste foram comparadas com a testemunha negativa (água destilada) e corrigidas pela fórmula de Abbott (ABBOTT, 1925). Com base nestas reduções no parasitismo, os pesticidas-teste foram classificados em quatro categorias, conforme a IOBC : 1 ) inócuo (<30\%); 2) levemente nocivo (30-79\%); 3) moderadamente nocivo (80-99\%); 4) nocivo (>99\%). Foram utilizadas quatro repetições para cada tratamento, sendo cada gaiola de exposição considerada uma unidade experimental. Os resultados obtidos, referentes à contagem do número de ovos parasitados por fêmea, foram testados quanto à normalidade da distribuição dos resíduos, sendo necessária a transformação para $\sqrt{\mathrm{X}}$. Posteriormente, os dados transformados foram submetidos à análise de variância e as médias dos tratamentos comparadas $(\mathrm{a}=0,05)$ pelo teste de Scott \& Knott (SCOTT \& KNOTT, 1974).

\section{RESULTADOS E DISCUSSÃO}

Bioensaio de ingestão em folhas de videira

O piretro natural foi o único inseticida que provocou mortalidade significativa de lagartas de $\boldsymbol{A}$. sphaleropa, resultando num controle de 47,78 e 55,56\%

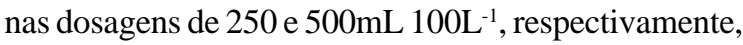
24 horas após a aplicação (HAA), sem haver diferenças significativas entre estas dosagens (Tabela 2). Neste mesmo período, as mortalidades observadas com as formulações de Natuneem ${ }^{\circledR}$ e Biopirol 7M ${ }^{\circledR}$ não diferiram da testemunha (Tabela 2). Às 48HAA, o piretro natural (250 e 500mL 100L ${ }^{-1}$ ) continuou apresentando ação sobre as lagartas tratadas, resultando em 53,41 e 64,77\% de controle, respectivamente (Tabela 2). Nesta avaliação, o Natuneem ${ }^{\circledR}$ e o Biopirol $7 \mathrm{M}^{\circledR}$, independentemebte da dosagem, continuaram apresentando mortalidade significativamente igual à da testemunha.

Nas avaliações realizadas às 72, $96 \mathrm{e}$ 120HAA, foi observado que o piretro natural apresentou um incremento na mortalidade do inseto, resultando num controle final de 77,65 e $85,88 \%$ nas

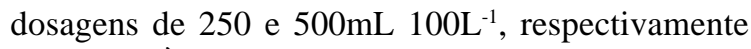
(Tabela 2). Às 120HAA, o Natuneem ${ }^{\circledR}$ e o Biopirol 7M ${ }^{\circledR}$ proporcionaram mortalidade de $16,47 \%$ e $11,76 \%$, respectivamente, na maior concentração $(500 \mathrm{~mL} 100$ $\mathrm{L}^{-1}$ ), sem, entretanto, diferir da testemunha.

Com relação ao nim, ALINIAZEE et al. (1997) avaliaram, em laboratório, a formulação Margosan $^{\circledR}$ (1000ppm de azadirachtina) e obtiveram 100\% de mortalidade de Archips rosanus (Lepidoptera: Tortricidae) às 48 HAA. Resultado similar foi observado por SUBAHARAN \& REGUPATHY (2000) com óleo de nim (1500ppm de azadirachtina) sobre Cydia

Tabela 2 - Número médio (X \pm EP) e mortalidade corrigida (entre parênteses) de lagartas de Argyrotaenia sphaleropa vivas quando alimentadas com discos de folhas de videira em laboratório (Temperatura $25 \pm 1^{\circ} \mathrm{C}$, UR $70 \pm 10$; Fotofase de 14 horas). Bento Gonçalves, RS, 2004.

\begin{tabular}{|c|c|c|c|c|c|c|}
\hline Produto & 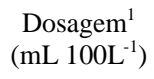 & $24 \mathrm{HAA}^{2}$ & 48 HAA & 72 HAA & 96 HAA & 120 HAA \\
\hline $\begin{array}{l}\text { Óleo de Nim } \\
\text { (Azadiracta indica) }\end{array}$ & 250 & $\begin{array}{c}9,00 \pm 0,0 \mathrm{a}^{3} \\
(0,00)^{4}\end{array}$ & $\begin{array}{c}8,80 \pm 0,13 \mathrm{a} \\
(0,00)\end{array}$ & $\begin{array}{c}8,70 \pm 0,21 \mathrm{a} \\
(0,00)\end{array}$ & $\begin{array}{c}7,90 \pm 0,28 \mathrm{a} \\
(7,06)\end{array}$ & $\begin{array}{c}7,90 \pm 0,23 a \\
(7,06)\end{array}$ \\
\hline $\begin{array}{l}\text { Óleo de Nim } \\
\text { (Azadiracta indica) }\end{array}$ & 500 & $\begin{array}{l}8,80 \pm 0,13 \mathrm{a} \\
\quad(2,22)\end{array}$ & $\begin{array}{c}8,60 \pm 0,16 \mathrm{a} \\
(2,27)\end{array}$ & $\begin{array}{c}8,40 \pm 0,27 a \\
(3,45)\end{array}$ & $\begin{array}{c}7,60 \pm 0,34 \mathrm{a} \\
(10,59)\end{array}$ & $\begin{array}{c}7,10 \pm 0,35 a \\
(16,47)\end{array}$ \\
\hline Piretro Natural & 250 & $\begin{array}{c}4,70 \pm 0,33 \mathrm{~b} \\
(47,78)\end{array}$ & $\begin{array}{c}4,10 \pm 0,48 b \\
(53,41)\end{array}$ & $\begin{array}{c}3,00 \pm 0,45 b \\
(65,52)\end{array}$ & $\begin{array}{c}2,70 \pm 0,52 b \\
(68,24)\end{array}$ & $\begin{array}{c}1,90 \pm 0,35 b \\
(77,65)\end{array}$ \\
\hline Piretro Natural & 500 & $\begin{array}{c}4,00 \pm 0,63 b \\
(55,56)\end{array}$ & $\begin{array}{c}3,10 \pm 0,48 b \\
(64,77)\end{array}$ & $\begin{array}{c}1,80 \pm 0,57 b \\
(79,31)\end{array}$ & $\begin{array}{c}1,50 \pm 0,50 b \\
(82,35)\end{array}$ & $\begin{array}{c}1,20 \pm 0,49 b \\
(85,88)\end{array}$ \\
\hline Ext. Pirolenhoso & 250 & $\begin{array}{l}8,90 \pm 0,10 \mathrm{a} \\
\quad(1,11)\end{array}$ & $\begin{array}{c}8,40 \pm 0,31 \mathrm{a} \\
(4,55)\end{array}$ & $\begin{array}{c}8,00 \pm 0,30 \mathrm{a} \\
(8,05)\end{array}$ & $\begin{array}{c}7,60 \pm 0,34 a \\
(10,59)\end{array}$ & $\begin{array}{c}7,30 \pm 0,42 \mathrm{a} \\
(14,12)\end{array}$ \\
\hline Ext. Pirolenhoso & 500 & $\begin{array}{c}8,90 \pm 0,10 \mathrm{a} \\
(1,11)\end{array}$ & $\begin{array}{c}8,60 \pm 0,16 \mathrm{a} \\
(2,27)\end{array}$ & $\begin{array}{c}8,20 \pm 0,20 \mathrm{a} \\
(5,75)\end{array}$ & $\begin{array}{c}7,70 \pm 0,30 \mathrm{a} \\
(9,41)\end{array}$ & $\begin{array}{c}7,50 \pm 0,34 \mathrm{a} \\
(11,76)\end{array}$ \\
\hline Testemunha & --- & $9,00 \pm 0,00 \mathrm{a}$ & $8,80 \pm 0,10 \mathrm{a}$ & $8,70 \pm 0,29 \mathrm{a}$ & $8,50 \pm 0,20 \mathrm{a}$ & $8,50 \pm 0,26 \mathrm{a}$ \\
\hline Média & & 7,61 & 7,21 & 6,62 & 6,17 & 5,84 \\
\hline CV (\%) & & 11,82 & 13,82 & 17,65 & 19,01 & 18,99 \\
\hline
\end{tabular}

${ }^{1} \mathrm{~mL}$ do produto comercial (p.c.) por 100L de água.

${ }^{2}$ Horas Após Aplicação (HAA).

${ }^{3}$ Médias não seguidas pela mesma letra na coluna diferem entre si pelo teste de Tukey $(\mathrm{P}=0,05)$.

${ }^{4}$ Mortalidade corrigida pela fórmula de Abbott (ABBOTT, 1925). 
leucostoma (Lepidoptera: Tortricidae), também observando $100 \%$ de mortalidade às 48HAA.

De maneira geral, os resultados obtidos por outros autores indicam que o nim é eficaz, principalmente, no controle de lepidópteros, sendo que insetos das ordens Coleoptera, Hemiptera e Orthoptera são menos sensíveis a azadirachtina (MORDUE \& NISBET, 2000; MARTINEZ, 2002). Em hipótese, a reduzida mortalidade obtida com a formulação do nim empregada nesse trabalho foi provavelmente devida à qualidade do produto comercial, pois a dosagem empregada foi equivalente ao avaliado para outras pragas da mesma família.

No Brasil, como os produtos à base de nim são comercializados sem registro oficial que informe a origem do produto e como não existe uma garantia quanto à padronização da concentração do ingrediente ativo, tal fato pode resultar em diferenças na eficácia das formulações comerciais, devendo ser observado pelos produtores antes do emprego do inseticida.

A baixa mortalidade das lagartas observada quando o Biopirol 7M ${ }^{\circledR}$ foi empregado pode ser devida, à aplicação do produto em laboratório sobre folhas destacadas das plantas. Segundo TSUZUKI et al. (2000), em condições de campo, o extrato pirolenhoso ativaria substâncias do metabolismo secundário, induzindo a resistência das plantas ao ataque dos insetos. Além disso, existem indícios de que características físicas e químicas, especialmente as quelatizantes presentes no extrato pirolenhoso, poderiam potencializar a eficiência de produtos fitossanitários e a absorção de nutrientes em pulverizações foliares (ZANETTI et al., 2003). Ainda, segundo esses mesmos autores, existe uma carência de informações experimentais a esse respeito, devendo ser avaliados em diferentes cultivos os benefícios do emprego do extrato pirolenhoso.

Bioensaios de seletividade em laboratório

O número médio de ovos parasitados por fêmea de T. pretiosum variou significativamente entre os pesticidas testados (Tabela 3). Na testemunha (água destilada), os valores obtidos foram de 42,12 ovos parasitados por fêmea, sendo que, na metodologia padronizada pela IOBC/WPRS com a espécie Trichogramma cacoeciae Marchal, um número mínimo de 15 ovos parasitados por fêmea é necessário para validar o experimento (HASSAN et al., 2000). Porém, para T. pretiosum, esse limiar inferior ainda não foi estabelecido.

Os inseticidas Natuneem ${ }^{\circledR}$, Biopirol $7 \mathrm{M}^{\circledR}$ e o Dipel $\mathrm{DF}^{\circledR}$ foram classificados como inócuos (classe 1), pois reduziram em menos de $30 \%$ o parasitismo das fêmeas de T. pretiosum (Tabela 3). Resultados similares foram obtidos por TAKADA et al. (2001), quando avaliaram o efeito de $B$. thuringiensis sobre Trichogramma dendrolimi Matsumura (Hymenoptera: Trichogrammatidae), demonstrando que a bactéria não afetou a emergência do parasitóide, quando aplicada

Tabela 3 - Efeito de inseticidas sobre a porcentagem de ovos parasitados por fêmea, redução (\%) na capacidade de parasitismo de Trichogramma pretiosum e classificação de toxicidade. Temperatura $25 \pm 1^{\circ} \mathrm{C}$; UR: $70 \pm 10 \%$; Fotofase: 14 horas. Pelotas, RS, 2004.

\begin{tabular}{|c|c|c|c|c|c|c|}
\hline Produto & $\begin{array}{c}\text { Nome } \\
\text { comercial }\end{array}$ & Dosagem $^{1}$ & Fêmeas/gaiola & $\begin{array}{c}\text { Ovos } \\
\text { parasitados/fêmea }^{2}\end{array}$ & $\mathrm{RP}^{3}(\%)$ & $\begin{array}{l}\text { Classe de } \\
\text { toxicidade }^{4}\end{array}$ \\
\hline Água destilada & ------ & ------ & $147,44 \pm 23,42$ & $42,12 \pm 6,63 \mathrm{a}$ & ------ & ------ \\
\hline $\begin{array}{l}\text { Óleo de Nim } \\
\text { (Azadirachta indica) }\end{array}$ & Natuneem ${ }^{\circledR}$ & 500 & $120,46 \pm 11,34$ & $47,75 \pm 5,57 \mathrm{a}$ & 0,00 & 1 \\
\hline Extrato Pirolenhoso & Biopirol 7M ${ }^{\circledR}$ & 500 & $126,39 \pm 6,09$ & $35,86 \pm 7,96 \mathrm{a}$ & 14,86 & 1 \\
\hline Bacillus thuringiensis & Dipel DF ${ }^{\circledR}$ & 100 & $167,87 \pm 11,54$ & $35,64 \pm 1,30 \mathrm{a}$ & 15,37 & 1 \\
\hline Piretro Natural & A definir & 250 & $165,07 \pm 20,54$ & $0,60 \pm 0,08 b$ & 98,58 & 3 \\
\hline Piretro Natural & A definir & 500 & $121,21 \pm 8,26$ & $0,00 \pm 0,00 \mathrm{~b}$ & 100,00 & 4 \\
\hline Fenthion & Lebaycid $500^{\circledR}$ & 100 & $151,28 \pm 11,71$ & $0,00 \pm 0,00 \mathrm{~b}$ & 100,00 & 4 \\
\hline
\end{tabular}

${ }^{1}$ Dosagem (g ou mL de produto comercial 100L).

${ }^{2}$ Médias ( \pm EP) não seguidas pela mesma letra diferem entre si pelo teste de Scott \& Knott a 5\% de probabilidade de erro.

${ }^{3} \mathrm{RP}=$ Redução na capacidade de parasitismo comparado com a testemunha.

${ }^{4}$ Classes da IOBC para teste de toxicidade inicial sobre adultos: 1=inócuo (<30\%), 2=levemente nocivo (30-79\%), 3=moderadamente nocivo (80-99\%), 4=nocivo (>99\%). 
sobre os ovos parasitados de Mamestra brassicae (Lepidoptera: Noctuidae).

Em laboratório, GONÇALVES-GERVÁSIO\&

VENDRAMIM (2004) avaliaram o efeito do tratamento de ovos de A. kuehniella com extrato de sementes de nim a $10 \%$ (1g do pó de semente de nim para cada $10 \mathrm{~mL}$ de água) sobre o parasitismo de T. pretiosum. Os ovos foram imersos antes e depois do parasitismo, sendo observado que o extrato afetou os insetos, reduzindo significativamente o número de ovos parasitados nas duas situações. Resultados similares foram observados por KLEMM \& SCHMUTTERER (1993) pulverizando ovos de Plutella xylostella (L.) com óleo de nim na concentração de $0,2 \%$. Os autores verificaram, em laboratório, uma redução do parasitismo de ovos por Trichogramma principium Sug. Et Sor. (Hymenoptera: Trichogrammatidae) e, no campo, por T. pretiosum. RAGURAN \& SINGH (1999), por sua vez, não observaram efeito deletério da aplicação de óleo de nim em concentração de 5,0mL 100L $\mathrm{L}^{-1}$ sobre ovos de Corcyra cephalonica (Stainton) (Lepidoptera: Pyralidae) parasitados por Trichogramma chilonis Ishii. Isso, em hipótese, pode indicar que produtos à base de nim em concentrações mais baixas e/ou através de diferentes formulações podem ser compatíveis com o uso de parasitóides do gênero Trichogramma.

O piretro natural, na menor dosagem avaliada (250mL $\left.100 \mathrm{~L}^{-1}\right)$, foi classificado como moderadamente nocivo (classe 3 ), reduzindo a capacidade de parasitismo de fêmeas de $\boldsymbol{T}$. pretiosum em 98,58\% (Tabela 3). A maior dosagem do piretro

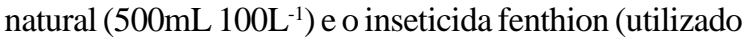
como inseticida padrão) foram classificados como nocivos (classe 4 ), reduzindo em $100 \%$ a capacidade de parasitismo de fêmeas (Tabela 3). Foi observado que, com a redução da dosagem do piretro natural, a ação tóxica sobre o parasitóide foi pouco minimizada. Nesse trabalho, os testes de toxicidade realizados com adultos de $\boldsymbol{T}$. pretiosum foram conduzidos em laboratório, representando a situação mais crítica para se avaliar um inseticida. Nesse caso, foi possível separar os produtos conforme seu efeito sobre a espécie $\boldsymbol{T}$. pretiosum, sendo que o Natuneem ${ }^{\circledR}$, o Biopirol 7M $\mathrm{M}^{\circledR} \mathrm{e}$ o Dipel DF ${ }^{\circledR}$ foram classificados como inócuos (Tabela 3). Segundo a metodologia da IOBC/WPRS, nesses casos não haveria necessidade de outros testes em casa de vegetação (semi-campo) ou a campo para avaliar a seletividade das formulações, pois se espera ali o mesmo comportamento dos parasitóides observado em laboratório. Entretanto, no caso do piretro natural e do fosforado fenthion, que foram classificados como moderamente nocivos e/ou nocivos, é fundamental que sejam conduzidos trabalhos em casa-de-vegetação e em vinhedos comerciais, para poder avaliar a persistência biológica e, conseqüentemente, estimar o risco de intoxicação dos inimigos naturais em condições de campo.

\section{CONCLUSÕES}

Os inseticidas Natuneem ${ }^{\circledR}$ e Biopirol 7M ${ }^{\circledR}$ (ambos a 250 e 500mL 100L ${ }^{-1}$ ) não causam mortalidade significativa de lagartas de Argyrotaenia sphaleropa quando aplicados sobre folhas de videira em laboratório, até 120 horas após o tratamento. O piretro natural (250 e 500mL 100L $\mathrm{L}^{-1}$ ) é eficiente no controle de lagartas ( $3^{\circ}$ ínstar) de $\boldsymbol{A}$. sphaleropa quando aplicado sobre folhas de videira em laboratório. Os inseticidas

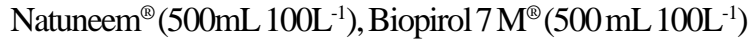

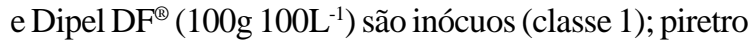

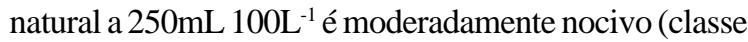
3), enquanto que, na dosagem de $500 \mathrm{~mL} 100 \mathrm{~L}^{-1}$, equivale-se ao inseticida fenthion (Lebaycid $500^{\circledR}$,

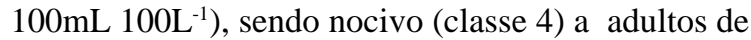
Trichogramma pretiosum em bioensaios conduzidos em laboratório.

\section{AGRADECIMENTOS E APRESENTAÇÃO}

À Coordenação de Aperfeiçoamento de Pessoal de Nível Superior (CAPES), pela concessão de bolsa ao pesquisador Morandi. Ao Conselho Nacional de Desenvolvimento Científico e Tecnológico (CNPq), pela concessão de bolsa aos pesquisadores Giolo, Manzoni, Botton e Grützmacher.

Parte da Dissertação de Mestrado do primeiro autor.

\section{REFERÊNCIAS}

ABBOTT, W.S. A method of computing the effectiveness of an insecticide. Journal of Economic Entomology, Lanham, v.18, p.265-267, 1925.

ALINIAZEE, M.T. et al. Laboratory and field evaluation of a neem insecticide against Archips rosanus L. (Lepidoptera: Tortricidae). Canadian Entomologist, Ontario, v.129, p.2733, 1997.

BASSO, C. et al. Eficacia de Trichogramma exiguum Pinto \& Platner y T. pretiosum Riley en el control de Argyrotaenia sphaleropa (Meyrick) y Bonagota cranaodes (Meyrick) en la vid en el Uruguay. Agrociência, Montevideo, v.3, n.1, p.2026, 1999.

BAVARESCO, A. Biologia, comportamento e controle das pragas do caquizeiro Argyrotaenia sphaleropa (Meyrick, 1909) (Lepidoptera: Tortricidae) e Hypocala andremona (Stoll, 1781) (Lepidoptera: Noctuidae). 2004. 110 f. Tese (Doutorado em Agronomia) - Curso de Pós-graduação em Fitossanidade, Faculdade de Agronomia Eliseu Maciel, Universidade Federal de Pelotas.

BENTANCOURT, C.M.; SCATONI, I.B. Lepidopteros de inportancia económica en el Uruguay (reconocimiento, 
biología y daños de las plagas agrícolas y forestales). [S.l.]: Hemisfério Sur - Facultad de Agronomia, 1995. V.1, 122p.

BIEZANKO, C.M. Olethreutidae, Tortricidae, Phalonidae, Aegeriidae, Glyphipterygidae, Yponomeutidae, Gelechiidae, Oecophoridae, Xylorictidae, Lithocolletidae, Cecidoseidae, Ridiaschinidae, Acrolophidae, Tineidae e Psychidae da zona sudeste do Rio Grande do Sul. Arquivos de Entomologia da Escola de Agronomia "Eliseu Maciel”, Série A, p.1-16, 1961.

BOTTON, M. et al. Ocorrência de Argyrotaenia sphaleropa (Meyrick) (Lepidoptera: Tortricidae) danificando pêssegos na Serra Gaúcha, Rio Grande do Sul. Neotropical Entomology, Londrina, v.32, p.503-505, 2003a.

BOTTON, M. et al. Manejo de pragas na cultura da videira. In: SEMINÁRIO INTERESTADUAL DE FRUTICULTURA, 3., 2003, Palmas. Anais... Palmas, PR: FACIPAL, 2003b. p.2331

ENCARNAÇÃO, F. Redução do impacto ambiental na produção de carvão vegetal e obtenção do ácido pirolenhoso como alternativa para proteção de plantas. Relato de experiência. Agroecologia e Desenvolvimento Rural Sustentável, Porto Alegre, v.2, n.4, p.20-23, 2001.

GONÇALVES-GERVÁSIO, R. de C.; VENDRAMIM, J.D. Efeito de extratos de Meliáceas sobre o parasitóide de ovos Trichogramma pretiosum Riley (Hymenoptera: Trichogrammatidae). Neotropical Entomology, Londrina, v.33, n.5, p.607-612, 2004.

HASSAN, S.A. et al. A laboratory method to evaluate the side effects of plant protection products on Trichogramma cacoeciae Marchal (Hym., Trichogrammatidae). In: CANDOLFI, M.P. et al. (Ed.). Guidelines to evaluate sideeffects of plant protection products to non-target arthropods. [S.1.]: IOBC/ WPRS, 2000. p.107-119.

KLEMM, U.; SCHMUTTERER, H. Effects of Neem preparations on Plutella xylostella L. and its natural enemies of the genus Trichogramma. Journal of Plant Diseases and Protection, Kasaragod, v.100, n.2, p.113-128, 1993.

KÖHLER, P. Tres nuevos microlepidópteros argentinos. Anales de la Sociedad Científica Argentína, Buenos Aires, v.128, p.369-374, 1939.

MANFREDI-COIMBRA, S. et al. Aspectos biológicos de Argyrotaenia sphaleropa (Meyrick, 1909) (Lepidoptera: Tortricidae) em dietas artificiais com diferentes fontes protéicas. Ciência Rural, Santa Maria, v.35, n.2, p.259-265, 2005.

MARTINEZ, S.S. O Nim: Azadirachta indica, natureza, usos múltiplos, produção. Londrina: IAPAR, 2002. 142p.

MEYRICK, E. Description of Microlepidoptera from Bolívia and Peru. Transactions of the Royal Entomological Society of London, London, v.15, p.13-43, 1909.
MIYASAKA, S. et al. Controle alternativo de pragas: fumaça e carvão como valiosas armas para a agricultura orgânica. Boletim Agro-Ecológico, Botucatu, v.3, n.14, p.17, 1999.

MORANDI FILHO, W.J. et al. Avaliação de inseticidas químicos e biológicos para o controle da lagarta-das-fruteiras Argyrotaenia sphaleropa (Meyrick, 1909) (Lep: Tortricidae). In: CONGRESSO BRASILEIRO DE FRUTICULTURA, 18., 2004, Florianópolis, SC. Resumos... Florianópolis: Epagri, 2004. 1 CD-ROM.

MORDUE, A.J.; NISBET, A.J. Azadirachtin from the neem tree Azadirachta indica: its action against insects. Anais da Sociedade Entomológica do Brasil, Londrina, v.29, n.4, p.615-632, 2000.

NORA, I.; SUGIURA, T. Estudo da entomofauna associada à cultura de pereiras japonesas (Housui, Kousui e Nijisseiki), em Santa Catarina, Brasil e técnicas de manejo. In: ENCONTRO NACIONAL DE FRUTICULTURA DE CLIMA TEMPERADO, 4., 2001, Fraiburgo, SC. Anais... Caçador: Epagri, 2001. p.164.

NUÑEZ, S. et al. Sex pheromone of South American tortricid moth Argyrotaenia sphaleropa. Journal of Chemical Ecology, New York, v.28, p.425-432, 2002.

RAGURAN, S.; SINGH, R.P. Biological effects of neem (Azadirachta indica) seed on an egg parasitoid, Trichogramma chilonis. Journal Economic Entomology, Lanham, v.92, p.1274-1280, 1999.

SCHMUTTERER, H. Properties and potential of natural pesticides from the Neem Tree, Azadirachta indica. Annual Review of Entomology, Palo Alto, v.35, p.271297, 1990.

SCOTT, A.J.; KNOTT, M.A. A cluster analysis method for grouping means in the analysis of variance. Biometrics, Arlington, v.30, p.507-512, 1974.

SUBAHARAN, K.; REGUPATHY, A. Neem based formulations for the management of tea flush worm Cydia leucostoma (Meyrick). Journal of Plantation Crops, Kasaragod, v.28, n.2, p.144-148, 2000.

TAKADA, Y. et al. Effects of various insecticides on the development of the egg parasitoid Trichogramma dendrolimi (Hymenoptera: Trichogrammatidae). Journal of Economical Entomology, Lanham, v.94, n.6, p.1340-1343, 2001.

TAMM, L. et al. Organic fruit production in humid climates of Europe: bottlenecks and new approaches in disease and pest control. Acta-Horticulturae, Leuven, v.638, p.333-339, 2004.

TSUZUKI, E. et al. Effect of chemical compounds in pyroligneous acid on root growth in rice plant. Japan Journal Crop Science, Tokyo, v.66, n.4, p.15-16, 2000.

ZANETTI, M. et al. nfluência do extrato pirolenhoso na calda de pulverização sobre o teor foliar de nutrientes em limoeiro "cravo". Revista Brasileira de Fruticultura, Jaboticabal, v.25, n.3, p.508-512, 2003. 